\title{
Scrub Typhus Presenting As Focal Neurological Deficit- A Rare Manifestation.
}

\author{
${ }^{1}$ Dr. Irappa Madabhavi, ${ }^{2}$ Dr. Guman Singh Negi, \\ ${ }^{3}$ Dr. Swaroop Revannasiddaiah, ${ }^{4}$ Dr. Mukesh Choudhary, ${ }^{5}$ Dr. Suhas Aagre, \\ ${ }^{6}$ Dr. Balwant Singh Negi, ${ }^{7}$ Dr. Mamta Lamba, ${ }^{8}$ Dr. Priyanka Das, \\ ${ }^{1}$ Resident, Department of Medical \& Pediatric oncology, GCRI, Ahmedabad, Gujarat, India. \\ ${ }^{2}$ Resident, Department of Internal Medicine, Indira Gandhi Medical College, Shimla, Himachal Pradesh, India. \\ ${ }^{3}$ Assistant Professor, Department of Radiotherapy \& Clinical Oncology, Swami Rama Cancer Hospital \& \\ Research, Institute, Haldwani, Uttarakhand, India. \\ ${ }^{4}$ Resident, Department of Medical \& Pediatric oncology, GCRI, Ahmedabad, Gujarat, India. \\ ${ }^{5}$ Resident, Department of Medical \& Pediatric oncology, GCRI, Ahmedabad, Gujarat, India. \\ ${ }^{6}$ Resident, Department of Surgery, Indira Gandhi Medical College, Shimla, Himachal Pradesh, India. \\ ${ }^{7}$ Resident, Department of Microbiology, SMS Jaipur, Jaipur, Rajasthan, India. \\ ${ }^{8}$ Resident, Department of Medical \& Pediatric oncology, GCRI, Ahmedabad, Gujarat, India.
}

\begin{abstract}
: 26 year old lady presenting with history of fever, altered sensorium, weakness of all the four limbs \& aphonia. On examination patient is semiconscious, aphonic, Upper Motor Neuron quadriparesis was present \& vitals were stable. Emergency Computer Tomography scan of head was done showing bilateral basal ganglia infarction. Cerebrospinal fluid analysis was showing normal study. Kidney \& Liver function tests were within normal range, blood, urine \& cerebrospinal fluid cultures were sterile. Work up for young stroke was within normal limit. MRI brain was done showing changes of Japanese encephalitis and serology for the same was negative. In view of epidemic of scrub typhus in sub-Himalayan regions IgM ELISA for scrub typhus was done \& was showing positivity for scrub typhus. Managed with Nasogastric tube feeding, Foleys catheterization, Injectable Azithromycin $500 \mathrm{mg} I V$ once a day for 5 days \& Doxycycline $100 \mathrm{mg}$ twice a day for 14 days, physiotherapy and frequent change of position. At discharge patient was fully conscious, was able to communicate with her attendants \& was able to walk with the help of attendants \& after 15 days of follow up patient was able to carry out day to day activities.
\end{abstract}

Key Words: Scrub typhus, focal neurological deficit, vasculitis, IgM Elisa, Azithromycin

\section{Introduction:}

Scrub Typhus or tsutsugamushi disease is a febrile illness caused by bacteria of the family Rickettsiaceae and named Orientia tsutsugamushi. Recently it has been found to be endemic in Subhimalayan region of India. Even though the neurological manifestations of scrub typhus is known entity, presenting as focal neurological deficit was unknown ${ }^{1,3}$ till now only one case was reported in the English Literature ${ }^{2}$. So we are reporting a case of scrub typhus presenting as Focal Neurological deficit.

\section{Case Report:}

26 year old lady, working in field, presented with history of fever, headache, body ache, myalgia, stupurous state, aphonic, weakness of all the four limbs, increased tone of all the 4 limbs \& altered sensorium. No history of similar complaints in the past. No history of seizures in the fast. No history of measles in the recent /past. No history of head injury/trauma in the past. No history suggestive neurocysticercosis or tuberculosis in the past. No history of any autoimmune or storage disorders in the patient or the family members. No history of malignancies in the past. No history of any drug intake or any poison intake in the past. No history of Coronary Artery Disease/Diabetes or Hypertension, No family history suggestive of young stroke, premature CAD, Diabetes or Hypertension. In view of endemicity of scrub typhus, residing in scrub typhus prevalent rural area \& unusual manifestation of the disease possibility of scrub typhus was kept.

In view of high index of clinical suspicion, patient was subjected to Weil-Felix, IgM ELISA for scrub typhus which was showing OX-K2 titers of 1:320 in weil-felix \& IgM Elisa for scrub typhus positivity. Computed Tomography Scan of brain shows presence of hypo dense areas involving the bilateral basal ganglia with CT attenuation value of $15-22 \mathrm{HU}$, possibility of encephalitis or infarct was kept.

Magnetic Resonance Imaging of brain shows altered signal intensity in areas involving the bilateral basal ganglia with lesion showing hyper intense on T1WI, T2WI \& FLAIR. No evidence of restriction seen on Diffusion Weighted images. There is also evidence of altered Signal Intensities in areas involving bilateral 
internal capsule, around the basal ganglia, bilateral medial temporal lobes and inferior frontal lobe of anterior part of left midbrain(substantia nigra). These areas shows hypointense on T1WI, hyper intense on T2WI, FLAIR. Overall findings are suggestive of Japanese Encephalitis. But IgM serology for Japanese encephalitis was negative.

Cerebrospinal fluid analysis, Random blood sugar, Kidney function tests, Liver function tests and Electrolytes were within normal limits. On examination there was no evidence of any raised Intracranial pressure features or meningeal signs. Serum parameters for random blood sugar /electrolytes /urea/creatinine/calcium/arterial blood gas analysis were normal. Chest radiography did not show any evidence of aspiration or infection.

After the diagnosis of scrub typhus patient was stabilized with Intra Venous lines, nasogastric feeding/Foleys catheterization. As per the standard of care for the scrub typhus she was initiated on azithromycin intravenously 500mg once a day for 5 days and doxycycline $100 \mathrm{mg}$ twice a day for 14 days through NG tube, I/V dextrose and thiamine was given. Patient showed improvement in GCS scale, kps scale, and motor power improvement. Initially in antigravity muscles followed by recovery of Sensory symptoms, bowel and bladder system improvement. Improvement was started on day $3^{\text {rd }}$ day of azithromycin, in view of clinical recovery azithromycin was continued for another 2 days.

At discharge patient was afebrile, was able to communicate with her attendants normally. Patient was discharged on day $14^{\text {th }}$ with complete recovery with no neurological deficit.

\section{Discussion:}

Increased prevalence of scrub typhus has been noted in the Asian subcontinents and tsutsugaushi triangle. It is endemic in certain geographic regions of India, Indonesia, Maldives, Mayanmar, Nepal, Srilanka and Thailand ${ }^{4-6 .}$ Scrub typhus is a small obligate gram negative, intracellular bacteria, Family rickettsiae, genus orientiae \& its Cell wall lacks peptidoglycans and lipopolysaccharides. Primary reservoir is a chigger/mite, larval stage, trombiculid mite (Leptotrombium daliense and others).Secondary reservoirs are mainly rodents \& humans. Incubation period is 5-20 days, mean10-12 days. Orientia tsutsugamushi is the causative agent \& transmitted to humans through the bite of thrombiculid mites. The mites have a four-stage lifecycle: egg, larva, nymph and adult. It is the accidental disease in humans \& chigger (larval) phase is the only stage that is parasitic on animals or humans ${ }^{7}$.

It divides and breeds within the phagocytes and escape from the cell back into the circulation to continue to proliferate on the endothelium of small blood vessels releasing cytokines which damage endothelial integrity, causing fluid leakage, platelet aggregation, polymorphs and monocyte proliferation, leading to focal occlusive end-angiitis causing micro infarcts. It is now well established that a majority of sequelae associated with human rickettsioses are the outcome of 'Rickettsial vasculitis' ${ }^{8}$. Especially affects skeletal muscles, skin, lungs, kidneys, brain and cardiac muscles. This can also cause venous thrombosis and peripheral gangrene.

Central nervous system manifestations are mainly due to endothelial cell damage, vasculitis , inflammatory cell infiltration, typhus nodules, new endothelial cell proliferation, thrombosis, ischemia \& infarction ${ }^{9}$ and intracranial hemorrhages are most likely due to vasculitis and perivasculitis accompanied by disseminated endothelial destruction in the capillaries caused by o.tsutsugamushi ${ }^{10}$.

Illness varies from mild and self limiting to fatal disease. Symptoms and signs vary in individuals with different strains. Commonest symptom high grade fevers, headache muscle pain, cough, and GI symptoms. Severe disease occurs in 2nd week with meningitis, meningo-encephalitis, deafness, pneumonia, ARDS, Multiorgan dysfunction \& myocarditis. Reinfection \& Relapses are seen due to variable immunity to different strains. Clinical signs are mainly rash, eschar, lympadenopathy, facial puffiness conjunctival suffusion, spleenomegaly, hepatospleenomegaly, pallor, icterus, ascites \& rarely focal neurological deficit. Diagnosis depends on the clinical, epidemiological, serological, isolation \& genetic assays ${ }^{11}$.

Treatment is with doxycycline $100 \mathrm{mg} 1 \mathrm{bd} /$ tetracycline $500 \mathrm{mg}$ qid/ chlormycetin $500 \mathrm{mg}$ qid/chloramphenicol for 7-15 days. Azithromycin 500mg 1OD for 3 days especially in pregnant women \& children. Rifampicin 600/900mg once a day for one week in resistant cases.

\section{Learning Points:}

1. Scrub typhus has varied manifestations varying from mild disease to fatal illness.

2. Focal neurological deficit is a rare manifestation of scrub typhus.

3. Good response to Doxycycline \& Azithromycin.

4. In resistant cases Rifampicin can be used. 


\section{References:}

[1]. Khachornsakdi Silpapojakul, MD; Chirapat Ukkachoke, MD; Suebsai Krisanapan, MD; Kamkarn Silpapojakul, MD. Rickettsial Meningitis and Encephalitis. Arch Intern Med. 1991; 151(9):1753-1757.

[2]. Dong-Eog Kim, MD; Seung-Hoon Lee, MD; Kyung-Il Park, MD; Kee-Hyun Chang, MD; Jae-Kyu Roh, MD.Scrub Typhus Encephalomyelitis With Prominent Focal Neurologic Signs .Arch Neurol. 2000;57:1770-1772.

[3]. Kim DM, Kim SW, Choi SH, Yun NR. Clinical and laboratory findings associated with severe scrub typhus. BMC Infect Dis 2010; 10:108.

[4]. Woodward TE, Osterman N. Rickettsial disease. In: Warren KS, Mahmoud AF, Eds, Tropical and Geographical Medicine. 2 Ed. New York: McGraw Hill, 1990; 918-30.

[5]. Goldsmith R, Heyneman D. Scrub typhus. In: Heyneman D, ed, Tropical Medicine and Parasitology. East Norwalk, Conn: Appleton \& Lange, 1989; 72-4.

[6]. Mcdade JE, Fishbein DB. Rickettsiae: The Rickettsiae. In: Behale PF ed CommWlicable and Infectious Disease: $8^{\text {th }}$ ed. St. Louis: Mosby, 1990; 864-90.

[7]. James MT. Mites and mite-borne disease. In: Harwood RF, ed, Medical Entomology. $6^{\text {th }}$ ed. New York: Macmillan, $1969 ; 379-87$.

[8]. Settle EB, Pinkerton H, Corbett AJ. A pathologic study of tsutsugamushi disease with notes on clinicopathologic correlation. J Lab Clin Med 1945;30:639-661.

[9]. Allen AC, Saitz S. A comparative study of the pathology of scrub typhus (tsutsugamushi disease) and other rickettsial disease. Am J Pathol 1945; 21:603-81.

[10]. Yang SH, L.S W, Liang CC, Ho YH, Chang ET, et al. (2005) Scrub typhus complicated by intracranial hemorrhage - A case report. Tzu Chi Med J 17: 111-114.

[11]. Shih-Hsien Yang, Lih-Shinn Wang1, Chung-Chao Liang, Yu-Huai Ho1, En-Ting Chang1, Chun-Heng Cheng.Am. J. Trop. Med. Hyg., 82(3), 2010, pp. 368-370. 